\title{
ENERGY INEQUALITIES FOR HYPERBOLIC EQUATIONS IN SEVERAL VARIABLES WITH MULTIPLE CHARACTERISTICS AND CONSTANT COEFFICIENTS( $\left.{ }^{(}\right)$
}

\author{
BY \\ GIDEON PEYSER
}

In this paper we shall derive energy integral inequalities for the linear hyperbolic partial differential equation of any order in several variables with multiple characteristics and constant coefficients.

In [1] Gårding uses an idea by Leray [6] to develop energy integrals for totally hyperbolic equations (including variable coefficients). Totally hyperbolic equations are subject to the severe restriction that all characteristics remain $\operatorname{distinct}\left({ }^{2}\right)$. In his method Garding multiplies the given operator of order $n$ by another operator of order $n-1$ whose characteristics separate those of the given operator. Using the Fourier transform and Parseval's formula he arrives at the energy inequality, which is an a priori estimate of the square integral of the solution of the given equation and all the derivatives up to order $n-1$. In our case of an equation with multiple characteristics we use a variant of Gårding's method. Our energy inequality is of a different form in as much as it provides an a priori estimate of the integral of the square of the solution and certain non-negative integral forms of its derivatives up to order $n-1$. However, we also show that for totally hyperbolic equations our energy inequalities reduce to the standard form.

In hyperbolic equations with multiple characteristics the influence of the lower order terms must be taken into account and hence the form of these lower order terms is suitably restricted. In totally hyperbolic equations no restrictions are imposed on the lower order terms. Our definition of hyperbolicity is therefore such that in the case of distinct characteristics no restrictions are imposed on the lower order terms. In [2] Gårding has given a criterion for hyperbolicity of equations in several variables with multiple characteristics and constant coefficients. A. Lax [4] extended this criterion, in the case of two independent variables, to include variable coefficients. We shall not be concerned here with

Presented to the Society, April 19, 1963 under the title Energy inequalities for hyperbolic equations with multiple characteristics; received by the editors September 7, 1962.

(1) This paper was supported by the National Science Foundation research grant NSFG21328.

(2) For a detailed exposition of totally hyperbolic equations we refer to Courant-Hilbert [5], in particular, pp. 661-676. 
the relationship between these criteria and our definition of hyperbolicity.

As an application of the energy integrals we solve in $\$ 3$ the Cauchy Problem for hyperbolic equations with multiple characteristics and constant coefficients using the Hilbert Space approach with the energy integrals as norm. Other approaches to this Cauchy Problem, independent of energy integrals were given by Courant-Lax [3] who utilize a reduction to two independent variables and an inversion of plane waves, and by Garding [2] with the aid of the Riesz operator.

In $\$ 4$ energy integrals are used to derive the differentiability of the solution of the Cauchy Problem under differentiability assumptions of the given functions.

1. Hyperbolic operators. Let $(t, x) \equiv\left(t, x_{1}, \cdots, x_{m}\right)$ denote $m+1$ real independent variables. We shall conveniently refer to $t$ as the time variable and to $x \equiv\left(x_{1}, \cdots, x_{m}\right)$ as the space variables.

Let

$$
E\left(\frac{\partial}{\partial t}, \frac{\partial}{\partial x}\right) \equiv E\left(\frac{\partial}{\partial t}, \frac{\partial}{\partial x_{1}}, \cdots, \frac{\partial}{\partial x_{m}}\right)
$$

be a differential operator of order $n$ with constant complex coefficients, such that $t=0$ is a noncharacteristic surface. We may therefore assume that the coefficient of $(\partial / \partial t)^{n}$ is 1 .

Let $E=H_{n}+L$ where $H_{n}$ is the principal part of $E$ and therefore homogeneous of order $n$ and $L$ an operator involving lower order derivatives. We now formulate conditions under which $E$ will be referred to as Properly Hyperbolic. The term "properly" refers to the fact that we permit multiple characteristics in which case the lower order terms are suitably restricted.

In the following we shall deal with polynomials in $\tau$ and with $\xi \equiv\left(\xi_{1}, \cdots, \xi_{m}\right)$ as parameters. Throughout the paper $\xi$ will denote a real vector. We shall also deal with the complex vector $s \xi$, $s$ a complex number. Factoring $H_{n}(\tau ; \xi)$, the principal part of $E$, we have

$$
H_{n}(\tau ; \xi)=\left(\tau-\lambda_{1}(\xi)\right) \cdots\left(\tau-\lambda_{n}(\xi)\right) .
$$

The $\lambda_{k}(\xi)$ are homogeneous of order one in $\xi$, i.e., for any complex number $s$,

$$
\lambda_{k}(s \xi)=s \lambda_{k}(\xi)
$$

The first requirement of a properly hyperbolic operator is that all $\lambda_{k}(\xi)$ are real for all real $\xi$. This implies, since the coefficient of $(\partial / \partial t)^{n}$ is 1 , that the coefficients of the principal part $H_{n}$ are all real. Note that we do not require that all $\lambda_{k}(\xi)$ are distinct nor do we require that if any two $\lambda_{k}(\xi)$ are equal for some $\xi$ they will be equal for all $\xi$. The only restriction this far is that the $\lambda_{k}(\xi)$ are real for all real $\xi$.

We consider the following $n+1$ polynomials $H_{n-i}(\tau ; \xi), i=0, \cdots, n$, which result from $n$ differentiations of $H_{n}(\tau ; \xi)$ with respect to $\tau$. 


$$
\begin{array}{rlr}
H_{n}(\tau ; \xi) & =\prod_{k=1}\left(\tau-\lambda_{k}^{n}(\xi)\right) ; & \lambda_{k}(\xi) \equiv \lambda_{k}(\xi) \\
H_{n-1}(\tau ; \xi) & =\frac{\partial}{\partial \tau} H_{n}(\tau ; \xi)=n \prod_{k=1}^{n-1}\left(\tau-\lambda_{k}^{n-1}(\xi)\right) \\
\quad \vdots & \\
H_{n-i}(\tau ; \xi) & =\frac{\partial}{\partial \tau} H_{n-i+1}(\tau ; \xi)=n(n-1) \cdots(n-i+1) \prod_{k=1}^{n-i}\left(\tau-\lambda_{k}^{n-i}(\xi)\right) \\
\quad \vdots & \\
H_{0}(\tau ; \xi) & =\frac{\partial}{\partial \tau} H_{1}(\tau ; \xi)=n ! .
\end{array}
$$

From elementary observations it follows that the roots of $H_{n-i}(\tau ; \xi)$ separate the roots of $H_{n-i+1}(\tau ; \xi)$ in the following sense: Listing the roots $\lambda_{j}^{n-i+1}(\xi)$, $j=1, \cdots, n-i+1$, and the roots $\lambda_{j}^{n-i}(\xi), j=1, \cdots, n-i$, in nondecreasing order (listing each root with its proper multiplicity), if $\lambda_{j}{ }^{n-i+1}<\lambda_{j+1}^{n-i+1}$ then $\lambda_{j}^{n-i+1}<\lambda_{j}^{n-i}<\lambda_{j+1}^{n-i+1}$ and if $\lambda_{j}^{n-i+1}=\lambda_{j+1}^{n-i+1}$ then $\lambda_{j}^{n-i}=\lambda_{j}^{n-i+1}$. From each polynomial $H_{n-i}(\tau ; \xi)$ we now create $n-i$ new polynomials $P_{n-i-1}^{k}(\tau ; \xi)$, $k=1, \cdots, n-i$, of degree $n-i-1$, by crossing out one factor at a time.

$$
P_{n-i-1}^{k}(\tau ; \xi)=n(n-1) \cdots(n-i+1) \prod_{j=1 ; j \neq k}^{n-i}\left(\tau-\lambda_{j}^{n-i}(\xi)\right) .
$$

From elementary considerations it follows that

$$
H_{n-i}=\sum_{k=1}^{n-i+1} P_{n-i}^{k}
$$

We now formulate the restriction on the lower order terms of a properly hyperbolic operator. If the lower order terms of $E$ are written as $L=L_{n-1}+\cdots+L_{0}$ where $L_{n-i}, i=1, \cdots, n$, are homogeneous operators of order $n-i$, then we require that the polynomial $L_{n-i}(\tau ; \xi)$ can be expressed as linear combinations of the polynomials $P_{n-i}^{k}(\tau ; \xi)$,

$$
L_{n-i}(\tau ; \xi)=\sum_{k=1}^{n-i+1} \gamma_{n-i}^{k}(\xi) P_{n-i}^{k}(\tau ; \xi) \quad i=1, \cdots, n
$$

with coefficients $\gamma_{n-i}^{k}(\xi)$ uniformly bounded for all real $\xi$.

We summarize our definition:

The operator $E \equiv H_{n}+L_{n-1}+\cdots+L_{0}$ is properly hyperbolic if

A. The roots $\lambda_{k}(\xi)$ of $(1)$ are all real for real $\xi$.

B. Each polynomial $L_{n-i}(\tau ; \xi)$ can be expressed as a linear combination of the polynomials $P_{n-i}^{k}, i=1, \cdots, n-i+1$.

C. The coefficients of the linear combinations of B, i.e., the $\gamma_{n-i}^{k}(\xi)$ of (5) are uniformly bounded for all real $\xi$. 
For later reference we note that from (5) it follows that

$$
L_{n-i}(\tau ; s \xi)=\sum_{k=1}^{n-i+1} \gamma_{n-i}^{k}(\xi) P_{n-i}^{k}(\tau ; s \xi), s \text { a complex number. }
$$

The following three nontrivial examples will serve to illuminate the meaning of proper hyperbolicity. In Example 1, condition B is violated. In Example 2, condition $\mathbf{C}$ is violated. The operator in Example 3 is properly hyperbolic.

EXAmple 1. Consider the operator:

$$
E=\left[\left(\frac{\partial}{\partial t}\right)^{2}-\left(\frac{\partial}{\partial x}\right)^{2}-\left(\frac{\partial}{\partial y}\right)^{2}\right]\left[\left(\frac{\partial}{\partial t}\right)^{2}-\left(\frac{\partial}{\partial x}\right)^{2}-2\left(\frac{\partial}{\partial y}\right)^{2}\right]+\left(\frac{\partial}{\partial t}-\frac{\partial}{\partial x}\right)^{3} .
$$

This operator does not satisfy condition B since the polynomial $\left(\tau-\xi_{1}\right)^{3}$ cannot be expressed as a linear combination of the polynomials:

$$
\begin{gathered}
\left(\tau-\sqrt{ }\left(\xi_{1}^{2}+\xi_{2}^{2}\right)\right)\left(\tau^{2}-\xi_{1}^{2}-2 \xi_{2}^{2}\right) \\
\left(\tau+\sqrt{ }\left(\xi_{1}^{2}+\xi_{2}^{2}\right)\right)\left(\tau^{2}-\xi_{1}^{2}-2 \xi_{2}^{2}\right) \\
\left(\tau^{2}-\xi_{1}^{2}-\xi_{2}^{2}\right)\left(\tau-\sqrt{ }\left(\xi_{1}^{2}+2 \xi_{2}^{2}\right)\right) \\
\left.\left.\left(\tau^{2}-\xi_{1}^{2}-\xi_{2}^{2}\right)(\tau+\sqrt{ }) \xi_{1}^{2}+2 \xi_{2}^{2}\right)\right) .
\end{gathered}
$$

This can be seen directly by taking $\xi_{1}=1, \xi_{2}=0$.

EXAMPLE 2(3).

$$
E=\left[\left(\frac{\partial}{\partial t}\right)^{2}-\left(\frac{\partial}{\partial x}\right)^{2}-\left(\frac{\partial}{\partial y}\right)^{2}\right]\left[\left(\frac{\partial}{\partial t}\right)^{2}-\left(\frac{\partial}{\partial x}\right)^{2}-2\left(\frac{\partial_{2}}{\partial y}\right)\right]+\left(\frac{\partial}{\partial x}\right)^{2} \frac{\partial}{\partial y}
$$

This operator violates condition $\mathrm{C}$. Indeed, for $\xi_{1} \neq 0, \xi_{2} \neq 0$ there exists a unique representation of $\xi_{1}^{2} \xi_{2}$ as a linear combination of the polynomials (7). Simple calculations show that the coefficient of the first polynomial is $\xi_{1}^{2} /\left(2 \xi_{2} \sqrt{ }\left(\xi_{1}^{2}+\xi_{2}^{2}\right)\right)$ which is clearly unbounded when $\xi_{2} \rightarrow 0$.

EXAMPLE 3.

$$
E=\left[\left(\frac{\partial}{\partial t}\right)^{2}-\left(\frac{\partial}{\partial x}\right)^{2}-\left(\frac{\partial}{\partial y}\right)^{2}\right]\left[\left(\frac{\partial}{\partial t}\right)^{2}-\left(\frac{\partial}{\partial x}\right)^{2}-2\left(\frac{\partial}{\partial y}\right)^{2}\right]+\frac{\partial}{\partial x}
$$

This operator is properly hyperbolic. Indeed, the roots of the characeristic polynomial are real for all real $\xi$. Hence A is satisfied. Furthermore, the following representation holds

$$
\xi_{1}=\frac{\xi_{1}}{\sqrt{ } 2\left(\frac{1}{2} \xi_{1}^{2}+\frac{1}{2} \xi_{2}^{2}\right)}\left[\left(\tau+\sqrt{ }\left(\frac{1}{3} \xi_{1}^{2}+\frac{1}{2} \xi_{2}^{2}\right)\right)-\left(\tau-\sqrt{ }\left(\frac{1}{3} \xi_{1}^{2}+\frac{1}{2} \xi_{2}^{2}\right)\right)\right] .
$$

(3) P. D. Lax used this operator in [3] for different, though related purposes. 
Since $\xi_{1} /\left(2 \sqrt{ }\left(\frac{1}{3} \xi_{1}^{2}+\frac{1}{2} \xi_{2}^{2}\right)\right)$ is clearly uniformly bounded for all real $\xi \neq 0$, it follows that $B$ and $C$ are satisfied.

To further justify our definition of proper hyperbolicity we have to show that a totally hyperbolic operator is also properly hyperbolic. A totally hyperbolic operator is such that the roots $\lambda_{k}(\xi)$ of (1) are real and distinct for all real $\xi \neq 0$, but no restrictions are imposed on the lower terms. We first note that in the case of a totally hyperbolic operator the polynomials $H_{n-i}(\tau ; \xi)$ each has $i$ distinct real roots $\lambda_{k}^{n-i}$ for real $\xi \neq 0$. Hence the polynomials $P_{n-i-1}^{k}(\tau ; \xi)$, $k=1, \cdots, n-i$, real $\xi \neq 0$, form sets of $n-i$ linearly independent polynomials of degree $n-i-1$ in $\tau$. Furthermore, these roots are homogeneous of order one in $\xi$, i.e., for any number $s, \lambda_{k}^{n-i}(s \xi)=s \lambda_{k}^{n-i}(\xi)$. Also, for all real $\xi$, with $|\xi| \equiv\left(\xi_{1}^{2}+\cdots+\xi_{m}^{2}\right)^{1 / 2}=1$, the numbers $\left|\lambda_{k}^{n-i}(\xi)\right|$ are uniformly bounded from above and the differences $\left|\lambda_{r}^{n-i}(\xi)-\lambda_{k}^{n-i}(\xi)\right|, r \neq k$, are uniformly bounded from below away from zero. Now suppose that $R_{n-i-1}(\partial / \partial t, \partial / \partial x)$ is any homogeneous operator of order $n-i-1$ with constant coefficients then

$$
\begin{aligned}
R_{n-i-1}(\tau ; \xi) & =\sum_{k=1}^{n-i} \delta_{k} P_{n-i-1}^{k}(\tau ; \xi), \\
\delta_{k} & =\frac{R_{n-i-1}\left(\lambda_{k}^{n-i}(\xi) ; \xi\right)}{P_{n-i-1}^{k}\left(\lambda_{k}^{n-i}(\xi) ; \xi\right)}=\frac{R_{n-i-1}\left(\lambda_{k}^{n-i}(\xi /|\xi|) ; \xi /|\xi|\right)}{P_{n-i-1}^{k}\left(\lambda_{k}^{n-i}(\xi /|\xi|) ; \xi /|\xi|\right)} .
\end{aligned}
$$

From the above considerations it follows that the coefficients $\delta_{k}$ are uniformly bounded for all real $\xi$, which completes the proof that a totally hyperbolic operator is also properly hyperbolic.

2. Energy integrals. The domain under consideration $V_{t_{0}}$ is the infinite slab $0 \leqq t \leqq t_{0}$ :

$V_{T}, T \leqq t_{0}$, will denote the slab $0 \leqq t \leqq T$.

$S_{T}$ will denote the hyperplane $t=T$.

A complex valued function in $V_{t_{0}}$ is called smooth if it possesses a sufficient number of continuous derivatives for the purpose at hand, and has bounded support in the space variables, i.e., vanishes for sufficiently large $|x| \equiv\left(x_{1}^{2}+\cdots+x_{m}^{2}\right)^{1 / 2}$. Throughout this paper $c$ will denote an unspecified constant depending only on the order and the coefficients of the given operator, on the number of independent variables and on the width $t_{0}$ of the domain $V_{t_{0}}$.

We define

$$
D^{\alpha} \equiv\left(\frac{\partial}{\partial t}\right)^{\alpha_{0}}\left(\frac{\partial}{\partial x_{1}}\right)^{\alpha_{1}} \cdots\left(\frac{\partial}{\partial x_{m}}\right)^{\alpha_{m}} ; \quad|\alpha| \equiv \alpha_{0}+\cdots+\alpha_{m}
$$

For specific derivatives we put $D_{0} \equiv(\partial / \partial t), D_{i} \equiv\left(\partial / \partial x_{i}\right), i=1, \cdots, m$. We shall make use of the following lemma:

LEMMA 2.1. If $|\alpha|=n,|\beta|=n-1$, and $f$ and $g$ are smooth functions, then 


$$
D^{\alpha} f D^{\beta} g+D^{\beta} f D^{\alpha} g=\sum_{j=0}^{m} D_{j} C_{\alpha \beta}^{j}(f, g)
$$

where $C_{\alpha \beta}$ are real symmetric forms in the derivatives of order $n-1$ of $f$ and $g$.

For a simple proof we refer to Gårding [1, pp. 74-75], also Courant-Hilbert [5, pp. 662-663].

We now consider the operators $H_{n-i}(D)$ corresponding to (2). Suppose

then

$$
\begin{aligned}
H_{n-i+1} & =\sum a_{\alpha} D^{\alpha} & |\alpha|=n-i+1, \\
H_{n-i} & =\sum b_{\beta} D^{\beta} & |\beta|=n-i,
\end{aligned}
$$

$$
\operatorname{Re} H_{n-i+1} f \overline{H_{n-i} f}=\frac{1}{2} \sum\left(a_{\alpha} D^{\alpha} \cdot f b_{\beta} D^{\beta} \bar{f}+a_{\alpha} D^{\alpha} \bar{f} \cdot b_{\beta} D^{\beta} f\right) .
$$

From Lemma 2.1 it follows that

$$
\operatorname{Re} H_{n-i+1} f \overline{H_{n-i} f}=\sum_{j=0}^{m} D_{j} A_{j}^{n-i}(f)
$$

where the $A_{j}^{n-i}(f)$ are Hermitian forms in the derivatives of order $n-i$ of $f$. The next two lemmas are central in this paper.

LEMMA 2.2. If $w$ is a smooth function, then

Proof. Let

$$
\int_{S_{T}} A_{0}^{n-i}(w) d x \geqq 0 \quad 1 \leqq i \leqq n, 0 \leqq T \leqq t_{0} .
$$

$$
A_{0}^{n-i}(w)=\sum a_{\alpha \beta}^{n-i} D^{\alpha} w D^{\beta} \bar{w} \quad|\alpha|=|\beta|=n-i
$$

and let $W$ be the Fourier transform of $w$

$$
W(T, \xi)=\int_{S_{T}} e^{-i x \xi} w(T, x) d x, \quad x \xi=x_{1} \xi_{1}+\cdots+x_{m} \xi_{m} .
$$

The Fourier transform of $D^{\alpha} w(T, x)$ is then

We denote

$$
W^{\alpha}(T, \xi)=D_{0}^{\alpha_{0}}\left(i \xi_{1}\right)^{\alpha_{1}} \cdots\left(i \xi_{m}\right)^{\alpha_{m}} W(T, \xi)
$$

$$
K^{n-i}(W)=\sum a_{\alpha \beta}^{n-i} W^{\alpha} \overline{W^{\beta}}
$$

From Plancherel's Theorem follows that the statement of the lemma is equivalent to showing that

$$
\int_{S_{T}} K^{n-i}(W) d \xi \geqq 0 .
$$

We substitute in (10) $f=W(t, \xi) e^{i x \xi}$. The expressions $A_{j}^{n-i}(f)$ are then clearly 
independent of $x$, and (10) can be written as

$$
\operatorname{Re} H_{n-i+1}\left(D_{0}, i \xi\right) W \overline{H_{n-i}\left(D_{0}, i \xi\right) W}=D_{0} K^{n-i}(W) .
$$

From (2) and (4) now follows, since $\lambda_{j}(i \xi)=i \lambda_{j}(\xi)$, that

$$
\begin{aligned}
D_{0} K^{n-}(W) & =\operatorname{Re} H_{n-i+1}\left(D_{0}, i \xi\right) W \sum_{k=1}^{n-i+1} \overline{P_{n-i}^{k}\left(D_{0}, i \xi\right) W} \\
& =\operatorname{Re} \sum_{k=1}^{n-i+1}\left(D_{0}-i \lambda_{k}^{n-i+1}(\xi)\right) P_{n-i}^{k}\left(D_{0}, i \xi\right) W \cdot \overline{P_{n-i}^{k}\left(D_{0}, i \xi\right) W} .
\end{aligned}
$$

Therefore

$$
K^{n-i}(W)=\frac{1}{2} \sum_{k=1}^{n-i+1}\left|P_{n-i}^{k}\left(D_{0}, i \xi\right) W\right|^{2}
$$

which is clearly non-negative. This establishes (12) and the lemma follows.

LEMMA 2.3. If the operator $E=H_{n}+L_{n-1}+\cdots+L_{0}$ is properly hyperbolic, then for a smooth function $w$

$$
\int_{S_{T}}\left|L_{n-i} w\right|^{2} d x \leqq c \int_{S_{T}} A_{0}^{n-i}(w) d x, \quad i=1, \cdots, n, 0 \leqq T \leqq t_{0} .
$$

Proof. The Fourier transform of $L_{n-i}(D) w$ is $L_{n-i}\left(D_{0}, i \xi\right) W$. From restriction $\mathrm{C}$ and (6) it follows that

$$
\left|L_{n-i}\left(D_{0}, i \xi\right) W\right|^{2} \leqq c \sum_{k=1}^{n-i+1}\left|P_{n-i}^{k}\left(D_{0}, i \xi\right) W\right|^{2} .
$$

Integrating both sides of (15) and using (13) it follows that

$$
\int_{S T}\left|L_{n-i}\left(D_{0}, i \xi\right) W\right|^{2} d \xi \leqq c \int_{S_{T}} K^{n-i}(W) d \xi .
$$

From Plancherel's Theorem applied to both sides of (16) follows our lemma. From (4) follows the

COROllary.

$$
\int_{S T}\left|H_{n-i} w\right|^{2} d x \leqq c \int_{S_{T}} A_{0}^{n-i}(w) d x \quad i=1, \cdots, n .
$$

We now formulate the energy inequality

THEOREM 2.1. If $E$ is properly hyperbolic and $w$ a smooth function satisfying the homogeneous data

$$
\left(\frac{\partial}{\partial t}\right)^{v} w(0, x)=0 \quad 0 \leqq v \leqq n-1,
$$

then there exists a constant $c$ such that 


$$
\sum_{i=1}^{n} \int_{S_{T}} A_{0}^{n-i}(w) d x \leqq c \iint_{V T}|E w|^{2} d t d x
$$

Proof. Integrating (10) over $V_{T}$ we get

$$
\int_{S_{T}} A_{0}^{n-t}(w) d x \leqq \frac{1}{2} \iint_{V_{T}}\left|H_{n-i+1} w\right|^{2} d t d x+\frac{1}{2} \iint_{V T}\left|H_{n-i} w\right|^{2} d t d x .
$$

Summing with respect to $i$ and using (17) we get

$$
\sum_{i=1}^{n} \int_{S_{T}} A_{0}^{n-i}(w) d x \leqq c \iint_{V_{T}}\left|H_{n} w\right|^{2} d t d x+c \sum_{i=1}^{n} \iint_{V_{T}} A_{0}^{n-i}(w) d t d x .
$$

Since $H_{n} w=E w-L_{n-1} w-\cdots-L_{0} w$ it follows from Lemma 2.2 that

$$
\sum_{i=1}^{n} \int_{S_{T}} A_{0}^{n-i}(w) d x \leqq c \iint_{V_{T}}|E w|^{2} d t d x+c \sum_{i=1}^{n} \iint_{V_{T}} A_{0}^{n-i}(w) d t d x
$$

From a well-known classical theorem now follows (19).

Since $A_{0}^{0}(w)=(n !)^{2}|w|^{2}$, we have the following

Corollary.

$$
\int_{S_{T}}|w|^{2} d x \leqq c \iint_{V_{T}}|E w|^{2} d t d x
$$

Integrating (19) with respect to $T$ from 0 to $t_{0}$ follows

THEOREM 2.2. If $w$ is smooth and satisfies the homogeneous data (18) then

$$
\sum_{i=1}^{n} \iint_{V_{t_{0}}} A_{0}^{n-i}(w) d t d x \leqq c \iint_{V_{t_{0}}}|E w|^{2} d t d x .
$$

Corollary.

$$
\iint_{V_{t_{0}}}|w|^{2} d t d x \leqq c \iint_{V_{t_{0}}}|E w|^{2} d t d x .
$$

In general Theorem 2.1 does not provide an a priori estimate for all the derivatives $D^{\alpha} w,|\alpha| \leqq n-1$. However, when the given operator is totally hyperbolic we have

THEOREM 2.2. If $E$ is totally hyperbolic with constant coefficients and if $w$ is a smooth function satisfying the homogeneous data (18) then

$$
\sum_{|\alpha| \leqq n-1} \int_{S_{T}}\left|D^{\alpha} w\right|^{2} d x \leqq c \int_{V T}|E w|^{2} d t d x .
$$

REMARK. This theorem is clearly not new, see Gårding [1] and CourantHilbert [5, p. 664]. However, it is included here to show that our energy 
inequalities for properly hyperbolic operators include the known energy inequalities for totally hyperbolic operators as a special case.

Proof. From (8) follows that for $|\alpha|=n-i$

$$
D_{0}^{\alpha_{0}} \xi_{1}^{\alpha_{1}} \cdots \xi_{m}^{\alpha_{m}}=\sum_{k=1}^{n-i} \delta_{k}(\xi) P_{n-i}^{k}\left(D_{0}, \xi\right)
$$

with $\delta_{k}(\xi)$ uniformly bounded for all real $\xi$. Denoting again by $W$ the Fourier transform of $w$ then it follows from (6) and (24) that

$$
\left|D_{0}^{\alpha_{0}}\left(i \xi_{1}\right)^{\alpha_{1}} \cdots\left(i \xi_{m}\right)^{\alpha_{m}} W\right|^{2} \leqq c \sum_{k=1}^{n-i+1}\left|P_{n-i}^{k}\left(D_{0}, i \xi\right) W\right|^{2}
$$

From (13) it follows that

$$
\left|D_{0}^{\alpha_{0}}\left(i \xi_{1}\right)^{\alpha_{1}} \cdots\left(i \xi_{m}\right)^{\alpha_{m}} W\right|^{2} \leqq c K^{n-i}(W) .
$$

Integrating (25) and applying Plancherel's Theorem we have

$$
\int_{S_{T}}\left|D^{\alpha} w\right|^{2} d x \leqq c \int_{S_{T}} A_{0}^{n-i}(w) d x \quad|\alpha|=n-i .
$$

From (19) now follows the theorem.

3. The Cauchy Problem for properly hyperbolic equations. We introduce the inner product of the smooth functions $v$ and $w$

and the norm

$$
(v, w)=\iint_{V_{t_{0}}} v \bar{w} d t d x
$$

$$
\|w\|^{2}=(w, w) .
$$

$H$ is the Hilbert space obtained by the completion of all smooth functions under the norm (27).

We now formulate the Cauchy Problem.

THEOREM 3.1. If $E$ is properly hyperbolic and $g \in H$, then there exists a unique strong solution $u \in H$ of

$$
E u=g
$$

with homogeneous data

$$
\left(\frac{\partial}{\partial t}\right)^{v} u(0, x)=0 \quad 0 \leqq v \leqq n-1,
$$

i.e., there exists a sequence of smooth functions $u^{(k)}$ such that

$$
\begin{aligned}
& \left\|u^{(k)}-u\right\| \rightarrow 0 \\
& \left\|E u^{(k)}-g\right\| \rightarrow 0
\end{aligned}
$$

and $(\partial / \partial t)^{v} u^{(k)}(0, x)=0, \quad 0 \leqq v \leqq n-1$. 
Proof. Uniqueness is a direct result of (22).

We prove the existence by using the projection theorem in Hilbert space. Let $K$ denote the subspace of $H$ consisting of all those functions $f \in H$ for which there exists a function $\omega \in H$ satisfying the homogeneous data (29) and the equation $E \omega=f$ in the strong sense, i.e., there exists a sequence of smooth functions $\omega^{(k)}$ such that

$$
\left\|\omega^{(k)}-\omega\right\| \rightarrow 0, \quad\left\|E \omega^{(k)}-f\right\| \rightarrow 0
$$

and $(\partial / \partial t)^{v} \omega^{(k)}(0, x)=0, \quad 0 \leqq v \leqq n-i$.

By virtue of (22) $K$ is a closed subspace of $H$. Inequality (22) continues to hold for all $\omega \in H$ satisfying the homogeneous data (29) and for which $E \omega$ exists in the strong sense. We shall show that the only element of $H$ perpendicular to the whole space $K$ is the zero element. It will follow that $K$ is the whole space $H$, which will then prove the existence.

Let $v \in H$ satisfy $(E w, v)=0$ for all smooth $w$ vanishing in a neighborhood of the surface $t=0$ (and therefore clearly satisfying the homogeneous data (29)).

We aim to show that $v=0$.

The properties of the following integral mollifiers are by now classic. (We shall need only some of their most elementary properties.) Let

$$
J_{\varepsilon} \omega=\frac{1}{\varepsilon^{m+1}} \iint_{V_{t_{0}}} j\left(\frac{t-\bar{t}+2 \varepsilon}{\varepsilon}, \frac{x-\bar{x}}{\varepsilon}\right) \omega(\bar{t}, \bar{x}) d \bar{t} d \bar{x}
$$

where $j(t, x)$ is an infinitely continuously differentiable function such that $j(t, x) \geqq 0 ; j(t, x)=0$ outside the cube $-1 \leqq t \leqq 1,-1 \leqq x_{i} \leqq 1$ and

$$
\iint_{-1 \leqq t, x \leqq 1} j(t, x) d t d x=1 .
$$

The adjoint mollifier is defined by

$$
J_{\varepsilon}^{*} \omega=\frac{1}{\varepsilon^{m+1}} \iint_{V_{t_{0}}} j\left(\frac{\bar{t}-t+2 \varepsilon}{\varepsilon}, \frac{\bar{x}-x}{\varepsilon}\right) \omega(\bar{t}, \bar{x}) d \bar{t} d \bar{x} .
$$

The following properties are well known:

If $\omega \in H$ then $J_{\varepsilon} \omega$ and $J_{\varepsilon}^{*} \omega$ are infinitely differentiable functions in $H$. In particular if $w$ is a smooth function then $J_{\varepsilon} w$ and $J_{\varepsilon}^{*} w$ are also smooth. $J_{\varepsilon} w$ vanishes in some neighborhood of $t=t_{0}$ and $J_{\varepsilon}^{*} \omega$ vanishes in some neighborhood of $t=0$.

We now have

$$
\begin{aligned}
\left(J_{\varepsilon} \omega, \sigma\right) & =\left(\omega, J_{\varepsilon}^{*} \sigma\right) \\
\left\|J_{\varepsilon} \omega-\omega\right\| & \rightarrow 0 \\
\left\|J_{\varepsilon}^{*} \omega-\omega\right\| & \rightarrow 0 \quad \text { as } \varepsilon \rightarrow 0 .
\end{aligned}
$$

$$
\left(E J_{\varepsilon}^{*} w, v\right)=0 \text { for all smooth } w .
$$


If $E=\sum a_{\alpha} D^{\alpha}$ then the adjoint operator $E^{*}=\Sigma(-1)^{|\alpha|} \bar{a}_{\alpha} D^{\alpha}$. From (32) follows, since the coefficients are constant, that

$$
\left(w, E^{*} J_{\varepsilon} v\right)=0 .
$$

Since the smooth functions $w$ are dense in $H$, it follows that $\left\|E^{*} J_{\varepsilon} v\right\|^{2}=0$.

Since $E$ is properly hyperbolic, so is $E^{*}$. We can therefore apply (22) to the operator $E^{*}$ replacing $t=0$ by $t=t_{0}$. Hence $\left\|J_{\varepsilon} v\right\|^{2} \leqq c\left\|E^{*} J_{\varepsilon} v\right\|^{2}=0$.

From $\varepsilon \rightarrow 0$ follows $v=0$ which completes the proof.

4. Differentiability of the solution. The differentiability of the solution of the Cauchy Problem depends on the differentiability of the right hand side of the equation. We shall derive in this section theorems regarding the differentiability of the solution with respect to all the independent variables. Several fine variations of these theorems are possible, in particular, differentiability with respect to some independent variables only. However, we shall not elaborate on this point.

This section is concerned with spaces of functions with strong (square integrable) derivatives whose definitions and properties are by now classic.

We shall also deal with vector functions $\left(\omega_{1}, \cdots, \omega_{m+1}\right)$ whose $m+1$ elements belong to $H$.

THEOREM 4.1. If $g \in H$ possesses in $V_{t_{0}}$ first order strong derivatives with respect to all the space variables $x$, then the solution of the Cauchy Problem (28) with homogeneous data (29) possesses in $V_{t_{0}}$ first order strong derivatives with respect to all the variables $t$ and $x$.

Proof. Let $w$ be a smooth function. We denote by $\hat{w}$ the following vector valued function

$$
\hat{w}=\left(w, w_{x_{1}}, \cdots, w_{x_{\ldots}}\right) .
$$

This means that the components of $\hat{w}$ consist of $w$ and all its $x$-derivatives.

The inner product and norm of these vectors are defined by

$$
\begin{aligned}
(\hat{v}, \hat{w}) & =\iint_{V t_{0}}\left(v \bar{w}+v_{x_{1}} \bar{w}_{x_{1}}+\cdots+v_{x_{m}} \bar{w}_{x_{m}}\right) d t d x, \\
\|\hat{w}\|^{2} & =(\hat{w}, \hat{w}) .
\end{aligned}
$$

$\hat{H}$ is the Hilbert space resulting from the completion of the vectors $\hat{w}$ with smooth elements under the norm (35).

It follows that for any vector $\hat{\omega} \in \hat{H}$ the first component is a function in $H$ possessing strong $x$-derivatives and the last $m$ components are these strong derivatives considered as functions in $H$.

The operator $E$ operating on smooth vectors $\hat{w}$ is defined by $E \hat{w}=$ $\left(E w, E w_{x_{1}}, \cdots, E w_{x_{m}}\right)$.

Since the coefficients of $E$ are constant it follows that 


$$
E \hat{w}=\left(E w,(E w)_{x_{1}}, \cdots,(E w)_{x_{m}}\right)
$$

and therefore $E \hat{w} \in \hat{H}$.

$\hat{K}$ will denote the subspace of $\hat{H}$ which consists of all vectors $\hat{f}$ for which there exists a vector $\hat{\omega} \in \hat{H}$ satisfying in the strong sense the equation

and the homogeneous data

$$
E \hat{\omega}=\hat{f}
$$

$$
\left(\frac{\partial}{\partial t}\right)^{\nu} \omega(0, x)=0 \quad 0 \leqq v \leqq n-1 .
$$

In other words, $f \in \hat{R}$ if and only if there exists a vector function $\hat{\omega} \in \hat{H}$ and a sequence of smooth vectors $\hat{\omega}^{(k)}$ such that

$$
\left\|\hat{\omega}^{(k)}-\hat{\omega}\right\| \rightarrow 0, \quad\left\|E \hat{\omega}^{(k)}-\hat{f}\right\| \rightarrow 0
$$

and $(\partial / \partial t)^{v} \hat{\omega}^{(k)}(0, x)=0,0 \leqq v \leqq n-1$. Applying (22) to each component of $E \hat{\omega}$ implies that $\hat{K}$ is a closed subspace of $\hat{H}$.

Similar to the procedure in $\$ 3$ we intend to show, using the projection theorem for Hilbert space, that $\hat{K}=\hat{H}$. We use the mollifiers operating on vector functions $\hat{\omega} \in \hat{H}$.

$$
\begin{aligned}
& J_{\varepsilon} \hat{\omega}=\left(J_{\varepsilon} \omega, J_{\varepsilon} \omega_{x_{1}}, \cdots, J_{\varepsilon} \omega_{x_{m}}\right), \\
& J_{\varepsilon}^{*} \hat{\omega}=\left(J_{\varepsilon}^{*} \omega, J_{\varepsilon}^{*} \omega_{x_{1}}, \cdots, J_{\varepsilon}^{*} \omega_{x_{m}}\right) .
\end{aligned}
$$

It is readily seen that the operators $J_{\varepsilon}$ and $J_{\varepsilon}^{*}$, operating on smooth functions $w$ commute with the space derivatives

$$
\begin{aligned}
& J_{\varepsilon} w_{x_{i}}=\left(J_{\varepsilon} w\right)_{x_{i}}, \\
& J_{\varepsilon}^{*} w_{x_{i}}=\left(J_{\varepsilon}^{*} w\right)_{x_{i}} .
\end{aligned}
$$

It follows that for any $\hat{\omega} \in \hat{H}$

$$
J_{\varepsilon} \hat{\omega}=\left(J_{\varepsilon} \omega,\left(J_{\varepsilon} \omega\right)_{x_{1}}, \cdots,\left(J_{\varepsilon} \omega\right)_{x_{m}}\right) .
$$

Therefore $J_{\varepsilon} \hat{\omega}$ and similarly $J_{\varepsilon}^{*} \hat{\omega}$ are infinitely differentiable vectors in $\hat{H}$, vanishing in a neighborhood of $t=t_{0}$ and $t=0$ respectively.

Let $\hat{v} \in \hat{H}$ be orthogonal to all vector functions of $\hat{K}$. In particular, since $J_{\varepsilon}^{*} \hat{w}$ is smooth for all smooth $\hat{w}$ and vanishes in a neighborhood of $t=0$, $\left(E J_{\varepsilon}^{*} \hat{w}, \hat{v}\right)=0$. Hence $\left(E J_{\varepsilon}^{*} \hat{w}, \hat{v}\right)=\left(\hat{w}, E^{*} J_{\varepsilon} \hat{v}\right)=0$.

Since the smooth vectors $\hat{w}$ are dense in $\hat{H}$, it follows that

$$
E^{*} J_{\varepsilon} \hat{0}=0 .
$$

Applying (22) to each component of (38), replacing $E$ by $E^{*}$ and $t=0$ by $t=t_{0}$, implies $J_{\varepsilon} \hat{v}=0$. From $\varepsilon \rightarrow 0$ follows $\hat{v}=0$.

This completes the proof that $\hat{K}=\hat{H}$. Therefore for the given $\hat{g} \in \hat{H}$ there exists 
a strong solution $\hat{u}$ of $E \hat{u}=\hat{g}$, satisfying the homogeneous data (37). But the first component of $\hat{u}$ is the (unique) solution of the Cauchy Problem $E u=g$ with homogeneous data (29). Since $u$ is the first component of $\hat{u} \in \hat{H}$ it possesses all first order strong $x$-derivatives. It remains to show the strong differentiability of $u$ with respect to $t$.

From (17) it follows that for $n>1$

$$
c \iint_{V_{t_{0}}} A_{0}^{l}(u) d t d x \geqq\left\|u_{t}\right\|^{2}-c \sum_{i=1}^{m}\left\|u_{x_{i}}\right\|^{2} .
$$

Hence $\left\|u_{t}\right\|^{2} \leqq c\left(\|g\|^{2}+\sum_{i=1}^{m}\left\|u_{x_{i}}\right\|^{2}\right)$, from which the differentiability of $u$ with respect to $t$ follows. If $n=1$ the differentiability of $u$ with respect to $t$ follows directly from the equation $E u \equiv u_{t}+\cdots=g$.

In a similar fashion follows the higher order differentiability theorem. We have to distinguish between differentiability up to order $n-1$ and differentiability of order higher than $n-1$.

THEOREM 4.2. (a) If $g \in H$ possesses all strong derivatives of the form

$$
D_{1}^{\alpha_{1}} \cdots D_{m}^{\alpha_{m}} \quad \alpha_{1}+\cdots+\alpha_{m} \leqq k, \quad k \leqq n-1
$$

then the strong solution of the properly hyperbolic equation $E u=g$ with homogeneous data (29), possesses all strong derivatives up to order $k$ with respect to all variables $t$ and $x$.

(b) If $g$ possesses all strong derivatives of the form

$$
D_{0}^{\alpha_{0}} D_{1}^{\alpha_{1}} \cdots D_{m}^{\alpha_{m}} \quad \alpha_{0} \leqq r, \alpha_{0}+\alpha_{1}+\cdots+\alpha_{m} \leqq r+n-1
$$

then $u$ possesses all strong derivatives up to order $r+n-1$.

\section{BIBLIOGRAPHY}

1. L. Gårding, Solution directe du Probléme de Cauchy pour les équation hyperboliques, Comptes rendus du colloque pour les équation aux déreivés partielles. Colloq. Internat., CNRS, Nancy, 1956, pp. 71-90.

2. - Linear hyperbolic partial differential equations with constant coefficients, Acta Math. 85 (1950), 1-62.

3. R. Courant and A. Lax, Remarks on Cauchy's problem for hyperbolic partial differential equations with constant coefficients in several independent variables, Comm. Pure Appl. Math. 8 (1955), 497-502.

4. A. Lax, On Cauchy's problem for partial differential equations with multiple characteristics, Comm. Pure Appl. Math. 9 (1956), 135-169.

5. R. Courant and D. Hilbert, Methods of mathematical physics, Vol. 2, Interscience, New York, 1962.

6. J. Leray, Hyperbolic differential equations, Lecture Notes, Institute for Advanced Study, Princeton, N.J., 1951-1952.

Pratt Institute,

BroOKLYN, New YoRK 